\title{
Aeromonas Pneumonia in a Trauma Patient Requiring Extracorporeal Membrane Oxygenation for Severe Acute Respiratory Distress Syndrome: Case Report and Literature Review
}

\author{
Nabil Issa and Lena M. Napolitano
}

\begin{abstract}
Background: Aeromonas species, particularly Aeromonas hydrophila, cause a wide spectrum of diseases in human being such as gastroenteritis; soft tissue infections including necrotizing fasciitis, meningitis, peritonitis, and bacteremia; but pneumonia and respiratory tract infections are uncommon.

Methods: Case report and literature review.

Results: A 30-year-old victim of a motor vehicle crash sustained pelvic fractures and splenic injury. Delayed splenic rupture caused sudden cardiorespiratory arrest. The patient was resuscitated but suffered septic shock and severe hypoxemia refractory to advanced mechanical ventilatory strategies. Aeromonas hydrophila was isolated as the causative pathogen of severe bilateral pneumonia. Venovenous extracorporeal membrane oxygenation (ECMO) was used temporarily. The patient recovered uneventfully.

Conclusion: This is the first case, to our knowledge, of the use of ECMO in a trauma patient with severe fulminant A. hydrophila pneumonia. Clinicians should be aware of the characteristics of this pathogen and associated clinical infections.
\end{abstract}

\begin{abstract}
$A$ EROMONAS SPECIES are water-borne motile gramnegative non-spore-forming bacilli widely distributed in the aquatic environment, both fresh- and salt-water. The species has been implicated in rare opportunistic infections in humans involved in aquatic trauma, those with burns, and immunocompromised patients such as those with leukemia or other cancer [1].

Aeromonas species, particularly Aeromonas hydrophila, cause a wide spectrum of diseases in humans such as gastroenteritis, soft tissue infections including necrotizing fasciitis, meningitis, peritonitis, and bacteremia [2-9]. Although there are reports of extraintestinal human infections caused by Aeromonas in both immunocompromised and immunocompetent patients, respiratory tract infections remain uncommon [10-12]. To our knowledge, the present paper is the first report of the use of extracorporeal membrane oxygenation (ECMO) support for severe acute respiratory distress syndrome (ARDS) related to Aeromonas pneumonia.

We report a patient involved in a motor vehicle crash who sustained multiple injuries and developed severe pneumonia
\end{abstract}

with worsening hypoxemia and ARDS requiring ECMO. Aeromonas hydrophila was the causative pathogen.

\section{Case Report}

A 30-year-old otherwise-healthy man was involved in a rollover motor vehicle crash as an unrestrained driver with ejection from the vehicle onto the pavement. The patient was neurologically and hemodynamically stable on the scene. He was airlifted to the University of Michigan Medical Level I Trauma Center. The initial examination confirmed hemodynamic stability, and the injuries included multiple pelvic fractures and a grade I splenic injury with no free fluid in the peritoneum by computed tomography (CT) imaging of the abdomen/pelvis. No thoracic injuries were identified by chest radiography (Fig. 1). He was admitted for non-operative management of his pelvic fractures and splenic injury.

On post-injury day 3 , he was found in cardiac arrest with pulselss electrical activity. The Advanced Cardiac Life Support protocol was initiated, with endotracheal intubation

Division of Acute Care Surgery, Department of Surgery, University of Michigan, Ann Arbor, Michigan. 


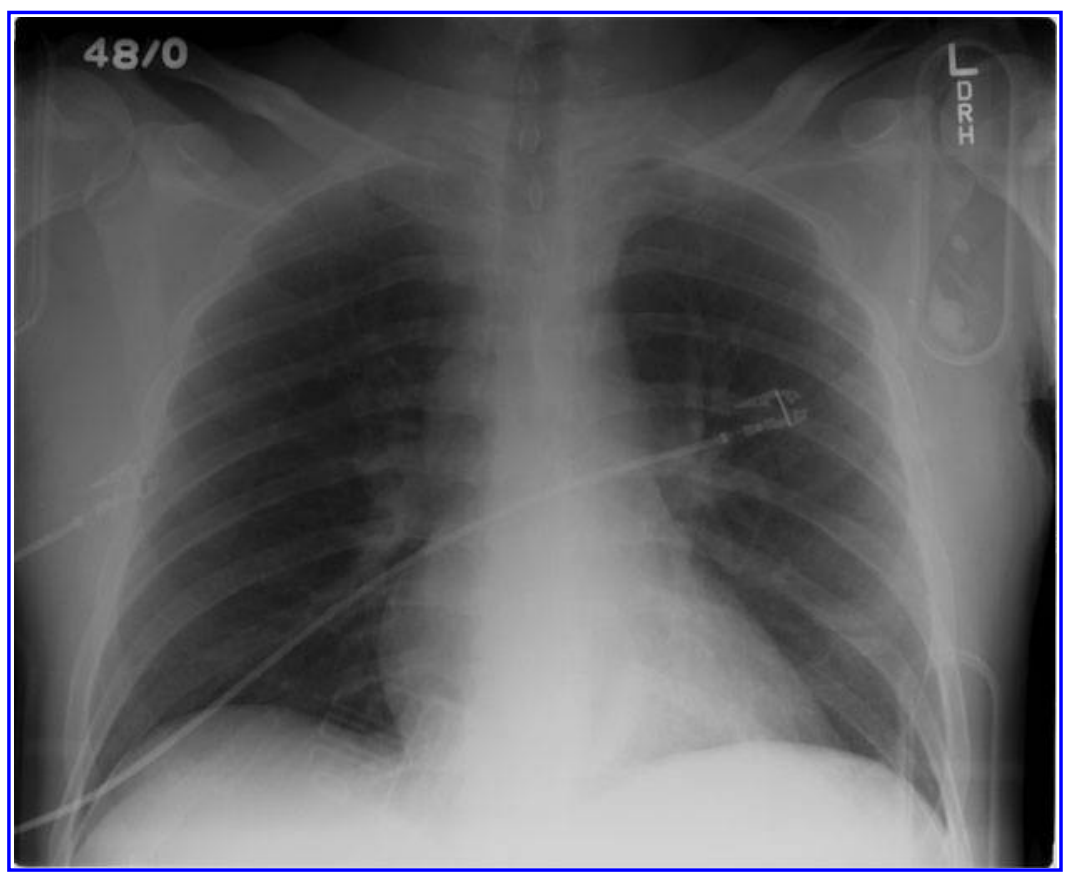

FIG 1. Chest radiograph on hospital admission post-accident revealing no traumatic injuries. Normal chest radiograph.

and resuscitation, leading to return of spontaneous cardiac rhythm and blood pressure, and he was transferred to the intensive care unit. He was intubated on first attempt, Mallampati Class I laryngoscopy view, with no evidence of aspiration. Delayed splenic rupture was confirmed by CT scan of the abdomen performed immediately after stabilization of hemodynamics (Fig. 2). Angiographic embolization was successful.

The patient subsequently developed severe hypoxemia with increasing oxygen requirement, and bilateral infiltrates were noted on the chest radiograph consistent with ARDS or bilateral bacterial or aspiration pneumonia (Fig. 3). A CT scan confirmed no evidence of pulmonary embolus. Evidence of hepatic ischemic injury with increased serum concentrations of his liver enzymes was noted on laboratory examination.

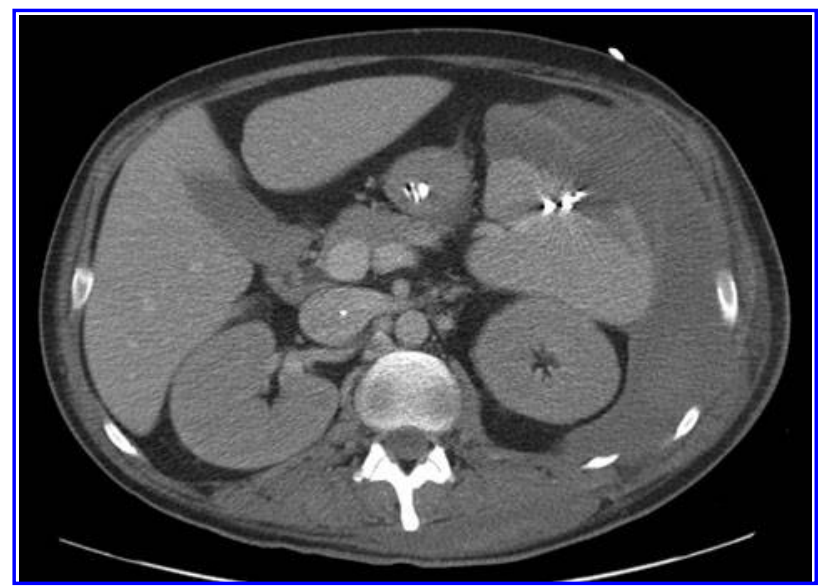

FIG. 2. Computed tomography scan of abdomen documenting splenic rupture, hemoperitoneum, and angiographic coil embolization.
Persistent fever and neutropenia developed, with a decrease in the white blood cell count to a nadir of $1.0 \times 10^{9} / \mathrm{dL}$. At this time, he had evidence of septic shock with multiple organ dysfunction syndrome, including acute renal failure necessitating renal replacement therapy. Cultures of blood and urine were obtained, and diagnostic bronchoscopy with bronchoalveolar lavage and quantitative cultures were performed to evaluate for pneumonia as a sepsis source. Empiric antimicrobial management with intravenous piperacillintazobactam and vancomycin was initiated. Vasopressors (norepinephrine, vasopressin) and steroids were used for management of his septic shock. Granulocyte colonystimulating factor (G-CSF) was given as treatment for severe neutropenia.

The patient's respiratory function continued to deteriorate, with persistent hypoxemia despite the provision of high $\mathrm{FIO}_{2}$ (1.0). Multiple mechanical ventilation strategies were used in attempts to improve oxygenation, including recruitment maneuvers, inverse-ratio pressure control ventilation, and high-frequency oscillatory ventilation (HFOV), but hypoxemia persisted.

An ECMO evaluation was requested for severe ARDS and refractory hypoxemia, and, given the likely reversible nature of his pulmonary disease and hypoxemia, he was cannulated for venovenous ECMO (VV-ECMO) with right internal jugular and right femoral cannulas. Full systemic anticoagulation with continuous infusion of unfractionated heparin was initiated for ECMO. Because he had undergone angiographic embolization of his splenic injury two days prior, it was recognized that he was at high risk for intra-abdominal bleeding, which would be difficult to diagnose. A diagnostic peritoneal lavage (DPL) catheter was inserted (open technique) after initiation of VV-ECMO to monitor for signs of intra-peritoneal bleeding while on ECMO and maintained sterile with an occlusive dressing. The catheter was interrogated daily by sterile saline lavage. 


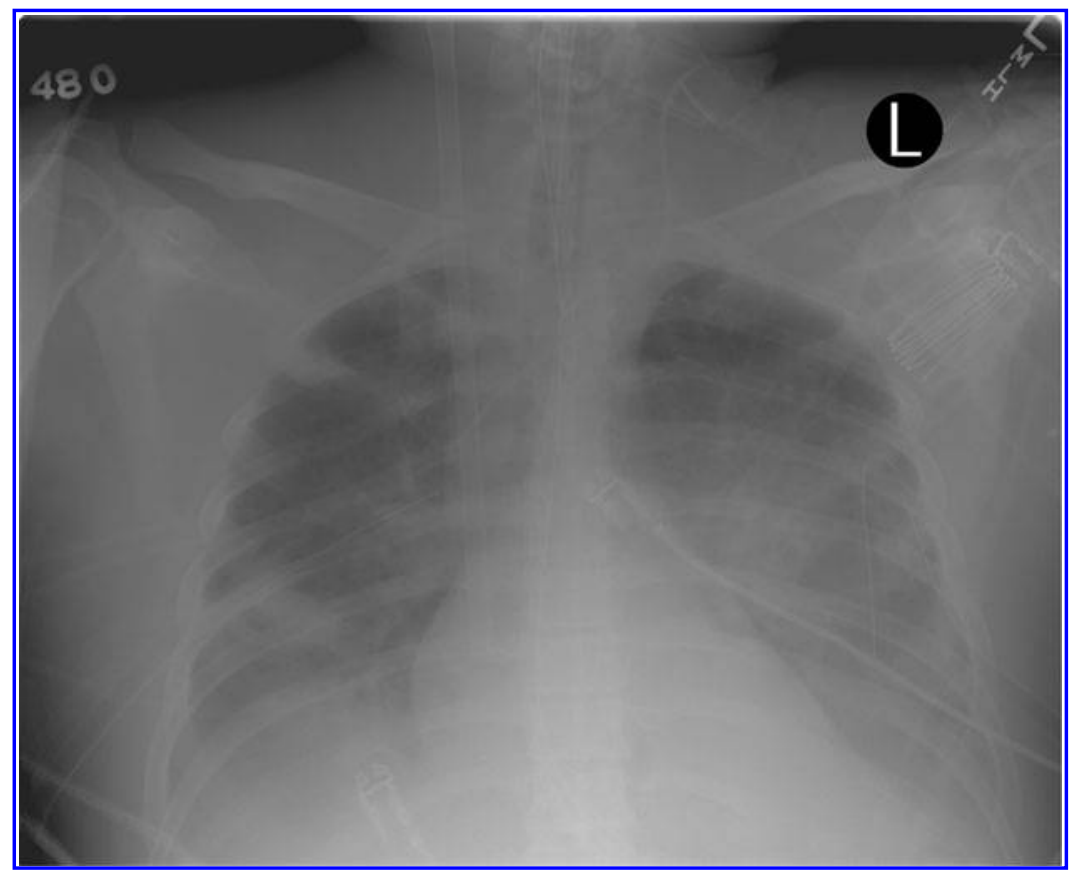

FIG. 3. Chest radiograph after cannulation for venovenous extracorporeal membrane oxygenation demonstrating bilateral infiltrates consistent with acute respiratory distress syndrome or bilateral pneumonia.

Cultures of bronchoalveolar lavage fluid confirmed Aeromonas hydrophila $>10,000$ colony-forming units (CFU) $/ \mathrm{mL}$ and Streptococcus pneumoniae $>10,000 \mathrm{CFU} / \mathrm{mL}$ (Table 1). All other cultures, including of blood, were negative. Levofloxacin was added, and the dose for piperacillin-tazobactam

Table 1. Antimicrobial Susceptibilities (Minimum Inhibitory Concentrations) of Pathogens Identified in Bronchoalveolar Lavage Fluid Cultures

\begin{tabular}{lcc}
\hline & $\begin{array}{c}\text { Aeromonas } \\
\text { hydrophila } \\
>10,000 \text { CFU/mL }\end{array}$ & $\begin{array}{c}\text { Streptococcus } \\
\text { pneumoniae } \\
>10,000 \text { CFU } / m L\end{array}$ \\
\hline Penicillin & $<8.00$ & $<0.06$ \\
Cefepime & $<8.00$ & \\
Piperacillin & $<8 / 4$ & \\
Piperacillin- & & \\
$\quad$ tazobactam & $<8.00$ & \\
Cefuroxime & $<8.00$ & \\
Cefoxitin & $<1.00$ & \\
Ceftriaxone & $<4.00$ & $<0.06$ \\
Aztreonam & $<4.00$ & \\
Meropenem & $<4.00$ & \\
Gentamicin & $<16.00$ & \\
Tobramycin & $<2 / 38$ & \\
Amikacin & & $<0.06$ \\
Trimethoprim- & & \\
$\quad$ sulfamethoxazole & & $<0.06$ \\
Erythromycin & $<.37$ \\
Clindamycin & $<1.00$ & $<0.125$ \\
Doxycycline & & $<0.50$ \\
Vancomycin & & \\
Levofloxacin & & \\
Ciprofloxacin & & \\
\hline
\end{tabular}

was adjusted according to the minimum inhibitory concentration (MIC) values on the microbiology report.

In anticipation of a prolonged need for ventilatory support, open tracheostomy was performed on VV-ECMO day two. The white blood cell count normalized, and G-CSF was discontinued. Hepatic enzymes normalized gradually. He was removed from ECMO after lung recruitment with HFOV. The ECMO was discontinued, and he was decannulated on postinjury day nine (VV-ECMO duration six days). An inferior vena cava (IVC) filter was placed just prior to decannulation with intravascular ultrasound guidance at the bedside in the surgical intensive care unit, with confirmation of IVC thrombus during the procedure. He was transitioned from HFOV to conventional ventilatory support with bilevel ventilation. He had full renal recovery, and renal replacement therapy was discontinued. Liberation from mechanical ventilation occurred on postinjury day 27, and his tracheostomy tube was removed (Fig. 4). The patient had a full uneventful recovery thereafter and was discharged home on postinjury day 51, able to perform all activities of daily living and with no neurologic sequelae from the cardiac arrest and severe hypoxemia.

\section{Discussion}

Aeromonas species is a rare causative agent of human infection. It is associated with high mortality rates, as high as $70 \%$ [13]. Aeromonas hydrophila is the most common species implicated in human infections, with rare reports of $A$. caviae and $A$. sobria causing infections of burn patients, also with high mortality [14]. Aeromonas hydrophila, a member of the family Vibrionaceae, is a gram-negative bacillus, a catalaseand oxidase-positive, facultative anaerobe that is ubiquitous; it is common in aquatic media and has a worldwide distribution. It is isolated frequently from water, soil, and certain 


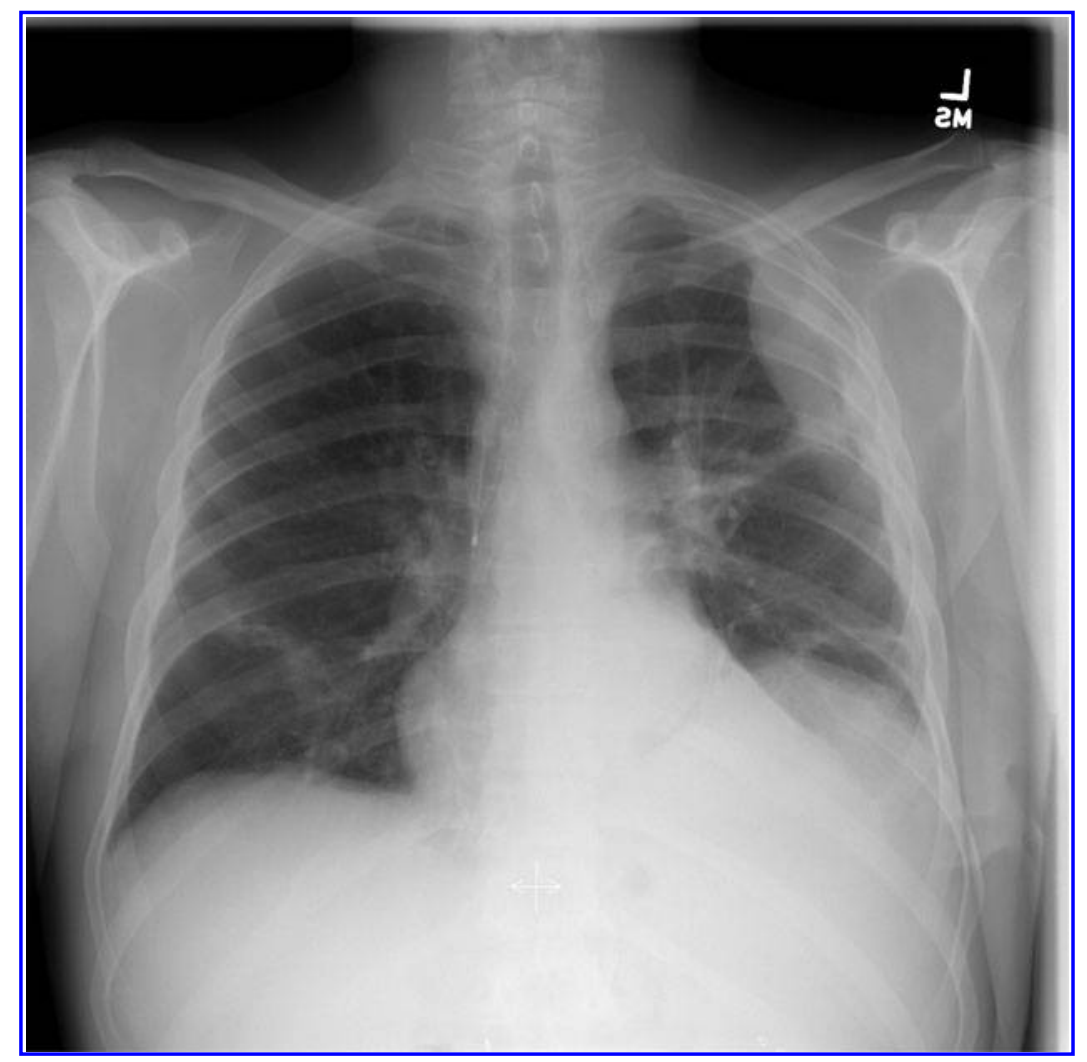

FIG. 4. Chest radiograph after extracorporeal membrane oxygenation decannulation, weaning from mechanical ventilation, and tracheostomy decannulation.

foods. In immunocompetent hosts, respiratory infection attributable to this pathogen occurs by ingestion of contaminated water [15].

Aeromonas hydrophila is generally susceptible to a wide range of antimicrobial drugs [16]. Aeromonas species can express $\beta$-lactamases and hence may be resistant to penicillin, first-generation cephalosporins, vancomycin, and clindamycin [17]. Despite the abundant knowledge about inducible, chromosomally-mediated $\beta$-lactamases among Aeromonas species, extended-spectrum beta-lactamase (ESBL)-producing A. hydrophila strains have rarely been isolated in clinical practice [18]. There are recent reports of A. hydrophila strains resistant to tetracyclines, cotrimoxazole, aminoglycosides, and extended-spectrum cephalosporins [19-21]. Apart from rare reports in the literature [22,23], A. hydrophila is consistently sensitive to third-generation cephalosporins, aztreonam, fluoroquinolones, aminoglycosides, and carbapenems [24-26].

Risk factors for Aeromonas infection include an immunocompromised state and an environmental water or soil source $[27,28]$. The gastrointestinal tract is the main portal of entry. Soft tissue infections, including necrotizing infections, have been reported in instances of injury and contamination of open wounds with soil [29,30]. Additional reports of sepsis in burn patients have documented contact with soil or water.

However, severe, even fatal, Aeromonas infections have been reported in immunocompetent normal children with pneumonia and sepsis [31,32]. Aeromonas also has been reported as a causative pathogen in cases of near-drowningassociated pneumonia [33].
The portal of entry of the Aeromonas organism was not readily identified in our patient, although aspiration may have occurred after ejection. Presumed aspiration pneumonia caused by Aeromonas hydrophila in immunocompetent patients has been described previously [34]. We also investigated the possibility that the patient acquired the infection through the hospital environment, either via the ventilator or as a cross-infection while in the trauma/burn ICU, but there were no reports of additional Aeromonas clinical infections in that ICU for the time period.

The severity of the A. hydrophila pneumonia infection in this patient necessitated the use of VV-ECMO as a bridging strategy to pulmonary recovery. The ECMO required full systemic anticoagulation with heparin, and, given the patient's underlying splenic injury, we utilized a DPL catheter as an inexpensive, reliable, and readily available monitoring device for signs of intraperitoneal bleeding, because transport of ECMO patients to a CT scanner can be challenging. This case highlights the potentially serious nature of Aeromonas pneumonia infection in trauma patients.

\section{Author Disclosure Statement}

No conflicting financial interests exist.

\section{References}

1. Murray PR, Baron EJ, Pfaller MA, et al. Manual of Clinical Microbiology. Edition 7. Washington, DC. ASM Press, 1999.

2. Semel JD, Trenholme G. Aeromonas hydrophila water-associated traumatic wound infections: A review. L Trauma 1990;30:324-332. 
3. Gold WL, Salit IE. Aeromonas hydrophila infections of skin and soft tissue: Report of 11 cases and review. Clin Infect Dis 1993;16:69-74.

4. Kelly KA, Koehler JM, Ashdown LR. Spectrum of extraintestinal disease due to Aeromonas species in tropical Queensland, Australia. Clin Infect Dis 1993;16:574-579.

5. Vukmir RB. Aeromonas hydrophila: Myofascial necrosis and sepsis. Intensive Care Med 1992;18:172-174.

6. Choi JP, Lee SO, Kwon $\mathrm{HH}$, et al. Clinical significance of spontaneous Aeromonas bacterial peritonitis in cirrhotic patients: A matched case-control study. Clin Infect Dis 2008;47:66-72.

7. Wu CJ, Lee HC, Chang TT, et al. Aeromonas spontaneous bacterial peritonitis: a highly fatal infectious disease in patients with advanced liver cirrhosis. J Formos Med Assoc 2009;108:293-300.

8. Ko WC, Lee HC, Chuang YC, et al. Clinical features and therapeutic implications of 104 episodes of monomicrobial Aeromonas bacteraemia. I Infect 2000;40:267-73.

9. Kim BN, Chung H, Shim TS. A case of spontaneous bacterial empyema and bacteremia caused by Aeromonas hydrophila. Eur J Clin Microbiol Infect Dis 2001;20:214-215.

10. Murata $\mathrm{H}$, Yoshimoto $\mathrm{H}$, Masuo $\mathrm{M}$, et al. Fulminant pneumonia due to Aeromonas hydrophila in a man with chronic renal failure and liver cirrhosis. Intern Med 2001;40:118-123.

11. Miyake $M$, Iga $K$, Izumi $C$, et al. Rapidly progressive pneumonia due to Aeromonas hydrophila shortly after neardrowning. Intern Med 2000;39:1128-1130.

12. Mukhopadhyay C, Bhargava A, Ayyagari A. Aeromonas hydrophila and aspiration pneumonia: A diverse presentation. Yonsei Med J 2003;44:1087-1090.

13. Janda JM, Duffey PS. Mesophilic aeromonads in human disease: Current taxonomy, laboratory identification and infectious disease spectrum. Rev Infect Dis 1988;10:980-997.

14. Lai CC, Shiao CC, Lu GD, Ding LW. Aeromonas hydrophila and Aeromonas sobria bacteremia: Rare pathogens of infection in a burn patient. Burns 2007;33:255-257.

15. Baddour LM, Baselski VS. Pneumonia due to Aeromonas hydrophila-complex: Epidemiologic, clinical, and microbiologic features. South Med J 1988;81:461-463.

16. Overman TL. Antimicrobial susceptibility of Aeromonas hydrophila. Antimicrob Agents Chemother 1980;17:612-614.

17. Ko WC, Lee HC, Chuang YC, Wu JJ. Clinical features and therapeutic implications of 104 episodes of monomicrobial Aeromonas bacteremia. J Infect 2000;40:267-273.

18. Ko WC, Wu HM, Chang TC, et al. Inducible $\beta$-lactam resistance in Aeromonas hydrophila: Therapeutic challenge for antimicrobial therapy. J Clin Microbiol 1998;36:3188-3192.

19. Ko WC, Yu KW, Liu CY, et al. Increasing antibiotic resistance in clinical isolates of Aeromonas strains in Taiwan. Antimicrob Agents Chemother 1996;40:1260-1262.

20. Fosse T, Giraud-Morin C, Madinier I, et al. Aeromonas hydrophila with plasmid-borne class A extended-spectrum $\beta$-lactamase TEM-24 and three chromosomal class B, C, and D $\beta$-lactamases, isolated from a patient with necrotizing fasciitis. Antimicrob Agents Chemother 2004;48:2342-2343.
21. Horii T, Morita M, Muramatsu H, et al. Antibiotic resistance in Aeromonas hydrophila and Vibrio alginolyticus isolated from a wound infection: A case report. J Trauma 2005;58:196200.

22. Balotescu C, Israil A, Radu R, et al. Aspects of constitutive and acquired antibioresistance in Aeromonas hydrophila strains isolated from water sources. Roum Arch Microbiol Immunol 2003;62:179-189.

23. Rodriguez CN, Campos R, Pastran B, et al. Sepsis due to extended-spectrum beta-lactamase-producing Aeromonas hydrophila in a pediatric patient with diarrhea and pneumonia. Clin Infect Dis 2005;41:421-422.

24. Skoll PJ, Donald AH, Simpson JA. Aeromonas hydrophila in burn patients. Burns 1998;24:350-353.

25. Barillo DJ, McManus AT, Cioffi WG, et al. Aeromonas bacteraemia in burn patients. Burns 1996;22:48-52.

26. Kienzle N, Muller M, Pegg S. Aeromonas wound infection in burns. Burns 2000;26:478-82.

27. Miyake $M$, Iga $K$, Izumi $C$, et al. Rapidly progressive pneumonia due to Aeromonas hydrophila shortly after neardrowning. Intern Med 2000;39:1128-1130.

28. Cigni A, Tomasi PA, Pais A, Cossellu S, Faedda R, Satta AE. Fatal Aeromonas hydrophila septicemia in a 16-year-old patient with thalassemia. I Pediatr Hematol Oncol 2003;25:674675.

29. Minnaganti VR, Patel Pj, Iancu D, et al. Necrotizing fasciitis caused by Aeromonas hydrophila. Heart Lung 2000;29:306308.

30. Lai CC, Ding LW, Hsueh PR. Wound infection and septic shock due to Aeromonas trota in a patient with liver cirrhosis. Clin Infect Dis 2007;44:1523-1524.

31. Kao HT, Huang YC, Lin TY. Fatal bacteremic pneumonia caused by Aeromonas hydrophila in a previously healthy child. J Microbiol Immunol Infect 2003;36:209-211.

32. Rodriguez CN, Campos R, Pastran B, et al. Sepsis due to extended-spectrum beta-lactamase-producing Aeromonas hydrophila in a pediatric patient with diarrhea and pneumonia. Clin Infect Dis 2005;41:421-422.

33. Ender PT, Dolan MJ, Dolan D, et al. Near-drowning-associated Aeromonas pneumonia. J Emerg Med 1996;14:737-741.

34. Mukhopadhyay C, Bhargava A, Ayyagari A. Aeromonas hydrophila and aspiration pneumonia: A diverse presentation. Yonsei Med J 2003;44:1087-1090.

Address correspondence to: Dr. Lena M. Napolitano

Division of Acute Care Surgery Department of Surgery University of Michigan Health System Room 1C421 University Hospital 1500 East Medical Dr. Ann Arbor, MI 48109-0033

E-mail: lenan@umich.edu 

This article has been cited by:

1. Kyle J Rehder, David A Turner, Ira M Cheifetz. 2011. Use of extracorporeal life support in adults with severe acute respiratory failure. Expert Review of Respiratory Medicine 5:5, 627-633. [CrossRef] 